\title{
Direct Measurement of Depletion Potentials in Mixtures of Colloids and Nonionic Polymers
}

\author{
D. Rudhardt, C. Bechinger, and P. Leiderer \\ Fakultät für Physik, Universität Konstanz, D-78457 Konstanz, Germany
}

(Received 17 February 1998)

\begin{abstract}
In colloidal suspensions containing a binary mixture of hard spheres depletion forces occur which substantially contribute to the interaction of the larger spheres among themselves and a wall, respectively. We investigated the depletion force acting on a large colloidal polystyrene sphere immersed in a solution of small, noncharged polymer coils close to a flat glass surface by means of total internal reflection microscopy. When the distance between the polystyrene sphere and the wall is smaller than the diameter of the polymer coils, an attractive potential acting on the sphere is observed which depends strongly on the polymer concentration. Our results are in agreement with theoretical predictions.

PACS numbers: 82.70.Dd, 36.20.-r, 64.75.+g
\end{abstract}

The stability of colloidal mixtures consisting of larger and smaller particles is well known to be strongly influenced by entropic depletion forces. Accordingly, those forces are essential in understanding phase separation phenomena and flocculation of binary hard-sphere mixtures, colloids in the presence of micelles, and of colloid polymer mixtures [1,2]. Very recently, it was suggested that depletion forces even may play an important role in the shape changes of phospholipid vesicles [3].

The principal phenomenon of depletion interaction is easily understood when, e.g., a hard sphere of radius $R$ suspended in a fluid containing smaller spheres of radius $r$ (the latter are referred to as macromolecules in the fol- lowing) in front of a wall at distance $z$ is considered (see Fig. 1). If $z$ decreases below the diameter of the macromolecules they are expelled from the region between the sphere and the wall. Consequently, the concentration of macromolecules becomes depleted in this region compared to that of the bulk, and an effective osmotic pressure causing a net attraction between the sphere and the wall occurs. Such an attractive force is also observed when the wall is replaced by another hard sphere.

The first quantitative explanation of this effect was given by Asakura and Oosawa [4]. According to their calculation the change in the Helmholtz free energy $\Delta F$ of a single sphere positioned at distance $z$ from a wall and suspended in a fluid of macromolecules can be written as

$$
\frac{\Delta F}{k_{B} T}=-n \pi\left[4 R r^{2}+\frac{4}{3} r^{3}+\frac{1}{3} z^{3}+(R-r) z^{2}-4 R r z\right] \Theta(2 r-z) \equiv \Phi_{\mathrm{dep} 1}(z)
$$

where $n$ is the concentration of the macromolecules and $\Theta$ is the Heaviside step function, respectively. Equation (1) is valid only for systems where the constituents can be considered as hard spheres, i.e., in systems which are driven solely by entropy. Because of this simple model the depletion force is predicted to be always attractive and nonzero only for distances $z<2 r$. It should be emphasized, however, that this theory is valid only in first order of $n$. In contrast, large deviations, e.g., even repulsive parts of the depletion potential, are predicted if correlation effects between macromolecules are taken into account $[5,6]$. The magnitude of depletion forces for small concentrations $n$ is typically on the order of nano- or piconewtons; therefore experimental techniques like surface forces apparatus [7], atomic force microscopy (AFM) [8], or total internal reflection microscopy [9] (the latter is also used in the present study and will be discussed in more detail below) are required for direct measurements of Eq. (1).

If long range interactions like electrostatic forces between the sphere and the macromolecules are additionally taken into account, Eq. (1) is no longer valid. According to a force balance model the magnitude and the range of the depletion forces are then predicted to be largely increased due to electrostatic repulsion [10]. In fact, most of the direct measurements of depletion forces were

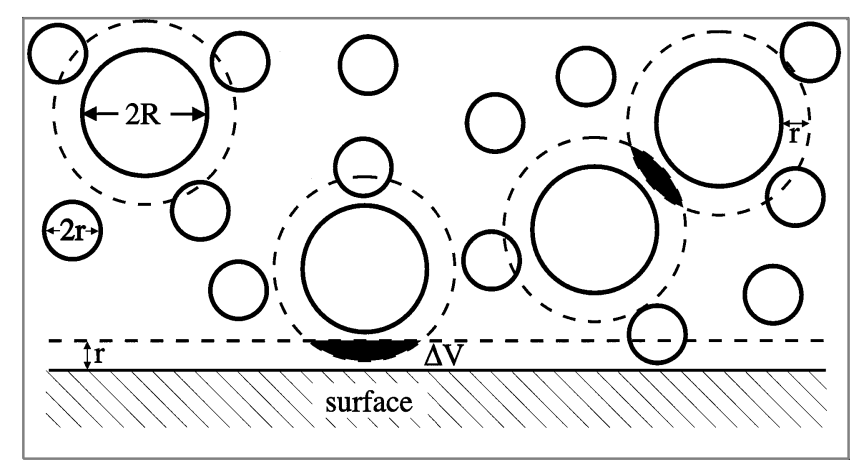

FIG. 1. Illustration of depletion interaction in a binary sphere mixture. The dashed regions correspond to areas not accessible to the centers of smaller spheres. 
performed on solutions of highly charged macromolecules like cetyltrimethylammonium bromide micelles [7], charged silica spheres [11], or polyelectrolytes [9].

In contrast to this, only a few experimental studies involving macromolecules with hard-sphere interaction are found in the literature. The entropic forces in binary hard-sphere systems have been studied with video microscopy [12] and laser radiation force techniques [13], respectively. Those measurements confirm the linear increase of the force acting on the larger particles when the concentration of macromolecules is increased, but the experiments do not provide the distance dependence of the interaction. In contrast to this, distance dependent measurements have been performed [8] by attaching a colloidal sphere to the cantilever of an AFM. It is not clear, however, to what amount this method (the sphere cannot be regarded as free) is intrusive to the investigated system.

In this paper we present the first systematic study of the depletion potential of a free colloidal sphere close to a wall in the presence of noncharged macromolecules. In contrast to previous work we investigated the dependence of the potential both on distance and on polymer concentration. We found that the potential is strongly affected by the concentration of macromolecules which was varied in our experiments.

We used total internal reflection microscopy (TIRM) [14] to measure the potential of a colloidal sphere immersed in a fluid of coiled, nonionic polymer chains close to a glass surface. When light is reflected at a solid/fluid interface above the angle of total internal reflection $\Theta_{C}$ (Fig. 2), an evanescent wave is formed which leaks into the fluid. The intensity of this evanescent wave decays exponentially perpendicular to the interface with a characteristic decay length $\beta^{-1}$ which depends on the incident angle $\Theta$, the wavelength of the light beam, and the refractive indices at the interface. When an object which scatters light, e.g., a colloidal sphere, approaches the surface close enough to enter the evanescent field frustrated total reflection will occur. The scattering intensity $I$ of the colloidal sphere is then proportional to that of the evanescent wave and can be written as $I \propto e^{-\beta z}[15]$. Measuring $I$ (which fluctuates due to Brownian motion of the sphere) as a function of time thus provides a sensitive and non-

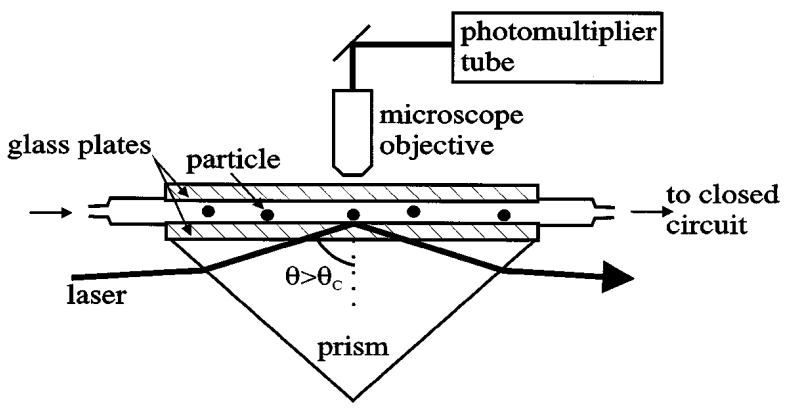

FIG. 2. Schematic TIRM setup used in our experiment. intrusive method to determine $z$. In thermal equilibrium the sphere-wall-interaction potential $\Phi(z)$ can be calculated as a function of $z$ by using the Boltzmann distribution $p(z)=e^{-\left[\Phi(z) / k_{B} T\right]}$. For details regarding TIRM and the data evaluation we refer to the literature (see, e.g., [14-16]).

Figure 2 shows a schematic representation of our TIRM setup. A cell was composed of two fused silica glass plates of $2 \mathrm{~mm}$ thickness each, separated by a spacer $(d=$ $1 \mathrm{~mm}$, not shown). After assembling the cell, a BK7 prism was optically matched to one of the glass plates. Then the cell was connected to a closed circuit which contained the colloidal suspension. We used monodisperse polystyrene (PS) spheres with radius $R=1.5 \mu \mathrm{m}$ which are suspended in water (IDC spheres). The particles are charge stabilized with sulfate surface groups. Only highly diluted suspensions with a particle density less than $0.5 \mathrm{~mm}^{-3}$ were used to guarantee that only a single particle was in the field of view and contributed to the scattering signal during our experiments. The suspension was pumped through the circuit which also contained an ion exchange resin and an electrical conductivity probe to control the ionic strength of the suspension. After adjusting the desired ionic strength a given amount of polyethylene oxide (PEO) with a molecular weight $M_{W}=2 \times 10^{6}$ was added. We chose PEO because it is one of the most extensively studied of all water-soluble nonionic and nonadsorbing synthetic polymers [17]. It is well known that below a critical concentration $n_{C}$ PEO forms coiled chains in water with a characteristic gyration radius $r_{G}$ which has been determined for the $M_{W}$ used in our experiments to $r_{G}=100 \mathrm{~nm}$ [18]. The maximum polymer concentration used in our experiments was $25.5 \mu \mathrm{m}^{-3}$ which is smaller than $n_{C}$ by a factor of 2.4. Therefore the polymer coils will be considered as hard-sphere-like macromolecules. The evanescent wave was obtained by reflecting a $\mathrm{HeNe}$ laser $(\lambda=632.8 \mathrm{~nm}, 10 \mathrm{~mW})$ from the glass/fluid interface at an angle $\Theta$ which corresponded to a penetration depth of $\beta^{-1}=0.845 \mu \mathrm{m}$. The measured potentials do not depend on the laser intensity which excludes the influence of light forces. No detectable change in the penetration depth and the electrical conductivity was observed after PEO was added to the suspension. The scattered intensity was collected by a microscope objective (Plan L50×, Leitz) and focused onto a photomultiplier tube. To obtain sufficient data and to minimize statistical errors the scattering intensity of a particle was sampled over at least $1000 \mathrm{~s}$ at a sampling rate of $50 \mathrm{~Hz}$. In the absence of depletion forces the potential of a negatively charged PS sphere above a glass plate is composed of two parts: towards larger distances $z$ the potential increases linearly, because the dominant force acting on the particle is due to gravity. At smaller $z$ the potential increases exponentially due to the electrostatic interaction between the negatively charged PS sphere and the fused silica surface which is also negatively charged when in contact with water [19]. In this case the potential acting on the sphere can be 
written as

$$
\frac{\Phi(z)}{k_{B} T}=B e^{-\kappa z}+\frac{G}{k_{B} T} z \equiv \Phi_{0}(z),
$$

where $B$ is a function of the surface potential of the plate and the sphere, respectively. $G=\left(\rho_{P}-\rho_{W}\right) V g$ is the weight of the particle with $\rho_{P}$ and $\rho_{W}$ being the density of the particle and water, $V$ the volume of the sphere, and $g$ the acceleration of gravity. This potential is experimentally confirmed by several groups using TIRM [14,16,20]. However, if polymer is added to the system, the potential between the sphere and the surface is considerably modified due to depletion forces as will be shown in the following.

Figures $3 a-3 c$ show typical scattering curves obtained from TIRM experiments at three different polymer concentrations. According to the above, high scattering intensities correspond to small distances $z$ between the PS sphere and the surface, whereas low intensities indicate large $z$. Figure 3 a corresponds to a polymer concentration $n=0$. The relatively large intensity fluctuations are characteristic for a particle in a broad potential well driven by Brownian motion. However, when some polymer $\left(n=7.6 \mu \mathrm{m}^{-3}\right)$ is added to the suspension, a qualitative different behavior is observed (Fig. 3b). Besides large intensity fluctuations we also find time intervals

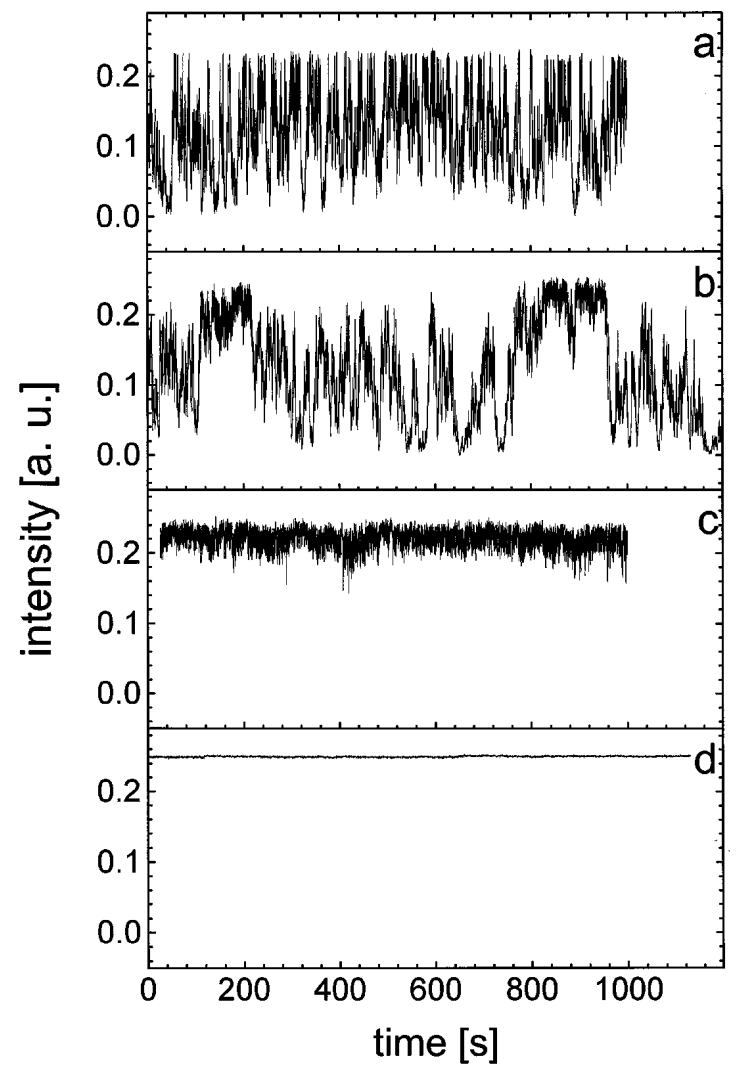

FIG. 3. Scattering intensity as a function of time for polymer concentrations $n=0$ (a), $n=7.6 \mu \mathrm{m}^{-3}$ (b), and $n=$ $25.5 \mu^{-3}$ (c). Irreversible sticking of the sphere (d). where $I$ is very high and varies only slightly with time. This finding which is very similar to the results of Kaplan et al. [12] indicates that there is an attractive, narrow potential well close to the surface where the sphere is trapped over several minutes. Increasing the polymer concentration further leads to a higher probability of finding the sphere in this trapped state until it is unable to escape from it above $n=10.2 \mu \mathrm{m}^{-3}$ (Fig. 3c). It is important to mention that even at the highest polymer concentrations used in our experiments, the PS spheres could be removed from the surface by gently pumping the suspension through the cell. The observed confinement of the PS particles to the surface is exactly what one would expect for a particle trapped in an entropic potential and will be analyzed in the following. Only if the salt concentration in our circuit is increased above $2 \mathrm{mM}$, irreversible sticking of the particles at the surface is observed which is attributed to van der Waals forces (Fig. 3d). The scattering intensity of such strongly sticking particles was assumed to correspond to $z=0$ and was used to obtain absolute particle-wall distances.

From raw data such as in Fig. 3, potential curves for different polymer concentrations were calculated. This is shown in Fig. 4 for $(a) n=0$, (b) $7.6 \mu \mathrm{m}^{-3}$, (c) $10.2 \mu \mathrm{m}^{-3}$, (d) $12.7 \mu \mathrm{m}^{-3}$, and (e) $25.5 \mu \mathrm{m}^{-3}$, respectively. The curves are separated in the vertical direction by $4 k_{B} T$ for clarity. As already mentioned, in the absence of macromolecules only gravity and electrostatic repulsion are acting on the particle giving rise to a relatively broad potential well. However, if a polymer

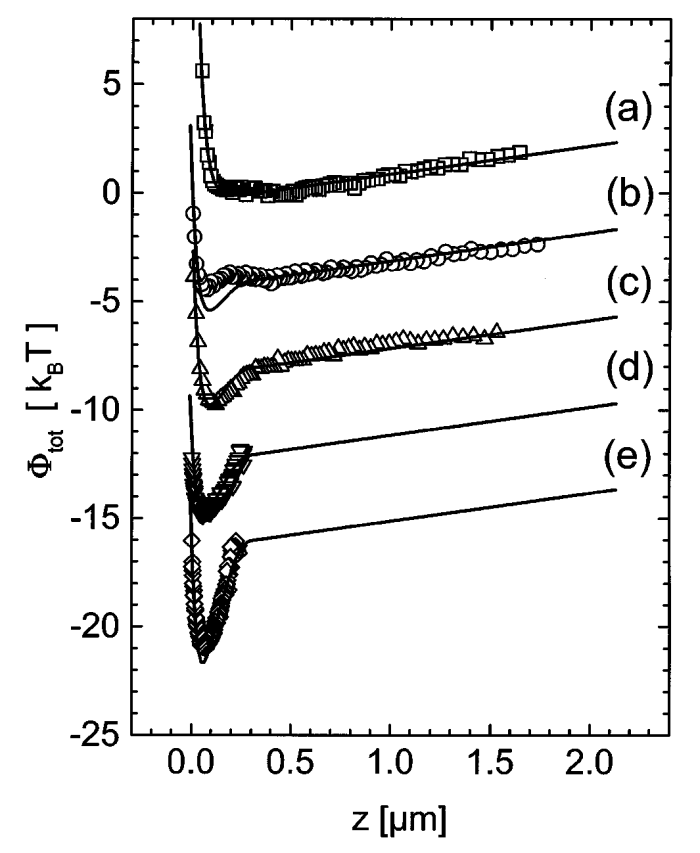

FIG. 4. Measured potential curves (symbols) of a PS sphere as a function of its distance $z$ from a flat surface for polymer concentrations $n=0(a), 7.6 \mu \mathrm{m}^{-3}(b), 10.2 \mu \mathrm{m}^{-3}$ $(c), 12.7 \mu \mathrm{m}^{-3}(d)$, and $25.5 \mu \mathrm{m}^{-3}(e)$. The solid lines are calculations according to Eq. (3). 
is added to the system (Figs. $4 b-4 e$ ) the potential is modified leading finally to a very pronounced potential well close to the surface. Since the depth of the potential well in Figs. $4 d$ and $4 e$ is about 3 to 5 times $k_{B} T$, the particle does not leave the well during our measuring time and therefore no data outside the well were collected.

To compare our results with theory, we first determined the exponential prefactor $B$ and the inverse screening length $\kappa^{-1}$ by fitting curve 3a using Eq. (2). We obtained values of $B=16.5$ and $\kappa^{-1}=33 \mathrm{~nm}$, the latter being in agreement with the ionic conductivity measurement. As mentioned above, $B$ and $\kappa^{-1}$ should not change when varying the polymer concentration; therefore both values were assumed to be constant when calculating the total potential

$$
\Phi_{\text {tot }}(z)=\Phi_{0}(z)+\Phi_{\text {depl }}(z) .
$$

The polymer concentration $n$ was determined before each experiment by weighing; the radius of the PS sphere was obtained by electron microscopy and found to be in agreement with the value provided by the manufacturer $(R=1.5 \mu \mathrm{m})$. Thus only one parameter, namely, the radius $r$ of the polymer, has to be determined. This was achieved by variation of $r$ in Eq. (3) until best agreement with all the experimental data in Fig. 4 was obtained. The solid lines in Fig. 4 show the calculated results corresponding to a polymer radius $r=150 \mathrm{~nm}$. As can be seen our data are in good agreement with what is predicted by Eq. (3).

The radius $r$ can be compared to the characteristic length scale of the PEO polymer, i.e., its radius of gyration $r_{G}$ which has been determined for different molecular weights [18]. For $M_{W}=2 \times 10^{6}$ a value of $r_{G}=101 \mathrm{~nm}$ is obtained. However, it is not clear whether $r$ has to be identical to $r_{G}$ because Eq. (1) is based on the assumption of rigid spheres. If the interaction of the polymer is not perfectly hard-sphere-like, e.g., due to steric interactions, $r$ is expected to be larger than $r_{G}$. This idea is also supported by the depletion measurements of Ohshima et al. [13] who used the same polymer in their experiments (PEO, $M_{W}=2 \times 10^{6}$ ). Their experimental values for the maximum depletion force are larger than expected if one assumes $r=r_{G}$. If we replot their results with $r=150 \mathrm{~nm}$, however, almost perfect agreement between theory and their data is obtained.

Finally we want to address the question to what extent higher order effects in the calculation of depletion forces have to be considered during our experiments. As has been shown by Mao et al. [5] a virial expansion yields a repulsive barrier at larger separations in addition to the well-known attractive force. This potential barrier is calculated as $\frac{W_{\max }}{k_{B} T}=1.2 n^{2} \frac{R}{r}$. Since for the $\frac{R}{r}$ ratio and the polymer concentrations used in our experiments $W_{\max }$ is less than $0.2 k_{B} T$ which is below the resolution limit of TIRM, higher order terms do not significantly contribute to our results and therefore justify a posteriori the assumption that the Asakura-Oosawa approximation is valid in our experiments.

In conclusion, we have measured the potential energy of a single polystyrene sphere suspended in a fluid of nonionic polymer in front of a wall using total internal reflection microscopy. We find the resulting potential to be strongly dependent on the polymer concentration which is a clear indication for the occurrence of depletion forces. The potential curves can be understood in terms of a simple theory using excluded volume arguments. No indications of repulsive depletion effects are found in our system. The radius for which best agreement between theory and data is obtained is in excellent agreement with the results of other authors.

This work was supported by the SFB 513 of the Deutsche Forschungsgemeinschaft.

[1] C. Cowell, F. K. Li-in-on, and B. Vincent, J. Chem. Soc. Faraday Trans. 74, 337 (1978).

[2] W.B. Russel, D. A. Saville, and W. R. Schowalter, Colloidal Dispersions (Cambridge University Press, Cambridge, England, 1989).

[3] A. D. Dinsmore, D. T. Wong, P. Nelson, and A. G. Yodh, Phys. Rev. Lett. 80, 409 (1998).

[4] S. Asakura and F. Oosawa, J. Chem. Phys. 22, 1255 (1954).

[5] Y. Mao, M. E. Cates, and H. N. W. Lekkerkerker, Physica (Amsterdam) 222A, 10 (1995).

[6] B. Götzelmann, R. Evans, and S. Dietrich, Phys. Rev. E 57, 6785 (1998).

[7] P. Richetti and P. Kekicheff, Phys. Rev. Lett. 68, 1951 (1992).

[8] A. Milling and S. Biggs, J. Colloid Interface Sci. 170, 604 (1995).

[9] A. Sharma, S. N. Tan, and J. Y. Walz, J. Colloid Interface Sci. 191, 236 (1997).

[10] J. Y. Walz and A. Sharma, J. Colloid Interface Sci. 168, 485 (1994).

[11] A. Sharma and J. Y. Walz, J. Chem. Soc. Faraday Trans. 92, 4997 (1996).

[12] P.D. Kaplan, L. P. Faucheux, and A. J. Libchaber, Phys. Rev. Lett. 73, 2793 (1994).

[13] Y. N. Ohshima, H. Sakagami, K. Okumoto, A. Tokoyoda, T. Igarashi, K. B. Shintaku, S. Toride, H. Sekino, K. Kabuto, and A. I. Nishio, Phys. Rev. Lett. 78, 3963 (1997).

[14] D. C. Prieve, F. Luo, and F. Lanni, Faraday Discuss. Chem. Soc. 83, 297 (1987).

[15] D. C. Prieve and J. Y. Walz, Appl. Opt. 32, 1629 (1993).

[16] S. G. Flicker and S. G. Bike, Langmuir 9, 257 (1993).

[17] E.A. Bekturov and Z.K. Bakauova, Synthetic WaterSoluble Polymers in Solution (Hüthig\&Wepf, New York, 1986), Chap. 3.

[18] K. Devanand and J.C. Selser, Macromolecules 24, 5943 (1991).

[19] R.J. Hunter, The Zeta Potential in Colloid Science (Academic, London, 1988), Chap. 7.

[20] D. Rudhardt, C. Bechinger, and P. Leiderer, Prog. Colloid Polym. Sci. (to be published). 